OPEN ACCESS

Edited by:

Hebao Yuan,

University of Michigan, United States

Reviewed by:

Honghao Zhang,

University of Michigan, United States

Hongwei Guo,

Guangxi Medical University, China

*Correspondence:

Dan-Dan Zhang

izhangdd@126.com

${ }^{\dagger}$ These authors contributed equally to this work

Specialty section:

This article was submitted to Pharmacology of Anti-Cancer Drugs,

a section of the journal

Frontiers in Pharmacology

Received: 27 January 2020 Accepted: 04 September 2020 Published: 25 September 2020

Citation:

Fang $T$, Jiang $Y-X$, Chen $L$, Huang $L$,

Tian $X-H$, Zhou $Y-D$, Nagle DG and

Zhang D-D (2020) Coix Seed Oil

Exerts an Anti-Triple-Negative Breast Cancer Effect by Disrupting miR-205/S1PR1 Axis.

Front. Pharmacol. 11:529962. doi: 10.3389/fphar.2020.529962

\section{Coix Seed Oil Exerts an Anti-Triple- Negative Breast Cancer Effect by Disrupting miR-205/S1PR1 Axis}

\author{
Ting Fang ${ }^{1,2+}$, Yi-Xin Jiang ${ }^{1+}$, Long Chen ${ }^{3+}$, Ling Huang ${ }^{1+}$, Xin-Hui Tian ${ }^{1}$, \\ Yu-Dong Zhou ${ }^{1,4}$, Dale G. Nagle ${ }^{1,5}$ and Dan-Dan Zhang ${ }^{1 *^{*}}$ \\ ${ }^{1}$ Interdisciplinary Integrative Medicine Research, Shanghai University of Traditional Chinese Medicine, Shanghai, China, \\ ${ }^{2}$ School of Pharmacy, Fujian University of Traditional Chinese Medicine, Fuzhou, China, ${ }^{3}$ Experiment Center for Science and \\ Technology, Shanghai University of Traditional Chinese Medicine, Shanghai, China, ${ }^{4}$ Department of Chemistry and \\ Biochemistry, College of Liberal Arts, University of Mississippi, University, Misissippi, MS, United States, ${ }^{5}$ Department of \\ BioMolecular Sciences and Research Institute of Pharmaceutical Sciences (RIPS), School of Pharmacy, University of \\ Mississippi, University, Mississippi, MS, United States
}

Coix Seed Oil (CSO) possesses a wide range of pharmacological activities. Kanglaite Injection, a commercial product of CSO, has been used clinically as an anticancer drug in China for decades. However, its molecular mechanisms on triple-negative breast cancer (TNBC) remains to be elucidated. In this study, the effect of CSO was evaluated on murine TNBC 4T1 cells and the orthotopic tumor-bearing mouse model and underlying mechanisms were explored. CSO suppressed cell proliferation, colony formation in vitro, and tumor growth in vivo. miR-205-5p was substantially altered in CSO treated tumor tissues compared to the control group by miRNA-sequencing analysis. Sphingomyelin metabolism (SM) decreased in serum in model group compared to the control group, while it increased by CSO administration by lipid metabolomics analysis. The expression of sphingosine 1 phosphate receptor 1 (S1PR1), the critical effector of SM, was downregulated upon CSO treatment. Mechanically, miRNA-205 directly targeted S1PR1 to regulate SM and cell proliferation. CSO reduced the expression of S1PR1, cyclinD1, and phosphorylation levels of STAT3, MAPK, and AKT while upregulated p27. These results revealed that CSO exerted an anti-TNBC effect via the miR-205/S1PR1 axis to regulate sphingomyelin metabolism, and the downstream STAT3/MAPK/AKT signal pathways were partly involved.

Keywords: Coix Seed Oil, triple-negative breast cancer, sphingomyelin metabolism, miR-205, Sphingosine-1phosphate receptors 1

\section{INTRODUCTION}

Breast cancer still incurs high morbidity and mortality globally and poses a severe health threat to women in recent years (Bray et al., 2018; Miller et al., 2019). Since triple-negative breast cancer (TNBC) lacks estrogen receptor (ER), progesterone receptor (PR), and human epidermal growth factor receptor (HER2), therapeutic options for TNBC are limited and leaving poor prognosis. 
TNBC is the most aggressive type with high clinical metastatic rates to bone, liver, brain, lung, and other organs in the late-stage of patients.

Currently, cytotoxic chemotherapy plays an essential role in treating TNBC patients with severe side effects, drug resistance, and poor patient compliance. Traditional Chinese Medicine (TCM) presents advantages including reducing toxicity and side effects, increasing the sensitivity of drugs, and improving immune responses in numerous cancers treatments.

Coix Seed Oil (CSO) is extracted from Coix seed (also named as Job's Tears seed or adlay), a kind of typical food and drug in China. Kanglaite Injection ${ }^{\circledR}$ (KLT) (Lu et al., 2008), a microemulsified injectable product derived from CSO, has been widely used to treat various tumors such as NSCLC (Duan, 2018; Wu et al., 2018), hepatocellular carcinoma (Yang et al., 2018), breast cancer (Guo et al., 2008), pancreatic (Liu et al., 2014; Schwartzberg et al., 2017) and gastric cancer (Zhang et al., 2017). KLT presented antineoplastic effect, reduced the side effects, and improved the quality of life such as cancer pain relief, cancer cachexia syndrome in patients (Liu et al., 2019; Zhang et al., 2019). Current pharmacological reports revealed KLT could induce apoptosis, block the cell cycle, inhibit angiogenesis, reverse multidrug resistance, and enhance the body's immune function via NF- $\mathrm{BB}$ and PI3K/Akt/mTOR signaling pathway (Liu et al., 2014) and regulation of oncomir hsa-miR-21 in serum (Wu et al., 2018). However, its exact antitumor mechanism, especially in TNBC, is still unclear.

In this study, we investigated the tumor-suppressive effect of CSO without excipient on TNBC and its potential mechanisms of action.

\section{MATERIALS AND METHODS}

\section{Cell Culture and Reagents}

The murine mammary carcinoma cell line 4T1-luc2 expressed luciferase was obtained from PerkinElmer. The human TNBC cell line MDA-MB-231 was obtained from the Type Culture Collection of the Chinese Academy of Sciences (Shanghai, China). Cells were maintained in DMEM medium (Gibco, Grand Island, NY, USA) supplemented with $10 \%$ fetal bovine serum (FBS; Gibco, Grand Island, NY, USA) at $37^{\circ} \mathrm{C}$ in a humidified environment (5\% $\mathrm{CO}_{2}$ and 95\% Air).

CSO (Batch Number:190109) was obtained from Zhejiang Kanglaite Pharmaceutical Co., Ltd. (Zhejiang, China). The 3[4,5-dimethylthiazol-2-yl]-2,5-diphenyltetrazolium bromide (MTT) and dimethyl sulfoxide (DMSO) were from SigmaAldrich (St. Louis, MO, USA). Antibodies against S1PR1 (55133-1-AP) and GAPDH (10494-1-AP) were purchased from Proteintech (Chicago, IL, USA). Antibodies against STAT3 (\#12640), p-STAT3 (\#9145), AKT (\#9272), p-AKT (\#13038), ERK1/2 (\#9102), p-ERK1/2 (\#9101), JNK (\#9252), pJNK (\#9251), P38 (\#9212), and p-P38 (\#9211) were obtained from Cell Signaling Technology (Boston, MA, USA). Antibodies against Cyclin D1 (ab134175) and p27 (ab32034) were obtained from Abcam (Cambridge, MA, USA). Trizol Reagent,
RNAIMAX reagent was purchased from Invitrogen (Carlsbad, CA, USA). MicroRNA primers, transcription kit, and universal PCR master mix, luciferase reporter plasmids, siRNA-S1PR1, siRNA-NC(negative control) miR-205-5p mimics, miRNA NC (negative control) were purchased from GenePharma (Shanghai, China). Dual-luciferase reporter assay kit was purchased from Promega (Madison, WI, USA)

\section{Quality Control of CSO by LC-MS}

High-resolution liquid-chromatography-mass spectrometry (LC-MS) was performed on Fisher Orbi-Trap Elite (Thermo, Waltham, MA, USA) to detect and analyze the main compounds in CSO.

\section{MTT Assay}

Exponentially grown $4 \mathrm{~T} 1$ cells were seeded at $2 \times 10^{3}$ cells per well into 96-well plates in a volume of $100 \mu \mathrm{l}$ and incubated under $37^{\circ} \mathrm{C}$ and $5 \% \mathrm{CO}_{2}$ condition overnight. Different concentrations of $\operatorname{CSO}(125,250,500$, and $1,000 \mu \mathrm{g} / \mathrm{ml}$, respectively) and $0.5 \%$ ethanol (as vehicle control) added and the incubation continued for another $72 \mathrm{~h}$. A stock solution of MTT $(5 \mathrm{mg} / \mathrm{ml})$ was added at $20 \mu \mathrm{l}$ per well. After $4 \mathrm{~h}$, the supernatant discarded and the precipitate was solubilized in DMSO (100 $\mu \mathrm{l} /$ well). The absorbance at $490 \mathrm{nM}$ measured by SpectraMax190 (Molecular Devices, CA, USA).

\section{Colony Formation Assay}

$4 \mathrm{~T} 1$ cells were seeded into 6 -well plates at the density of 300 cells per well and allow to attach overnight and then cultured with different concentrations of CSO. After $24 \mathrm{~h}$, the medium of different groups was replaced with complete medium. The medium of each well was discarded and washed after 7 days. Cells were fixed, washed, and stained with crystal violet $(0.5 \%)$. The colonies with over 50 cells were imaged and accounted.

\section{Orthotopic Tumor Bearing Mouse Model}

Female BALB/c mice aged 5 weeks were purchased from the Shanghai Slack Laboratory Animals Co., Ltd and housed at the Shanghai University of Traditional Chinese Medicine animal facility. Murine $4 \mathrm{~T} 1$ cells $\left(1 \times 10^{6}\right)$ were injected into the upper mammary fat pad of mice. After the implantation of cells 7 days, these tumor-bearing mice were randomly divided into the model group and CSO treatment group. Mice of the model group only received vehicle control ( $4 \%$ egg yolks) with daily gavage. The CSO treatment group was administered $3 \mathrm{ml} \mathrm{CSO} / \mathrm{kg} / \mathrm{d}$ in egg yolks suspension with daily gavage. The volume of tumors was measured daily using a Vernier caliper and calculated using the formula $\mathrm{V}=\left(\mathrm{ab} \mathrm{b}^{2}\right) / 2$ (the longest diameter $\mathrm{a}$, the shortest diameter b). These mice were weighed and measured the volume of tumors daily. After 14 days of continuous vehicle control or CSO administration, these mice were sacrificed to gain the blood and tumor of each mouse. Another control group (six mice) administered vehicle control was set for the blood samples.

Female BALB/c nude mice aged 5-6 weeks (weight: 18-22 g) were purchased from the Shanghai Slack Laboratory Animals Co., Ltd and housed at the Shanghai University of Traditional Chinese Medicine animal facility. Human TNBC MDA-MB-231 cells were 
resuspended at a density of $1 \times 10^{8}$ cells/ml. Five million cells $(50 \mu \mathrm{l})$ of mixed Matrigel $(50 \mu \mathrm{l})$ were injected into the second mammary fat pad of mice. When the tumors volume of these mice reached around $200 \mathrm{~mm}^{3}$, the mice were randomly divided into the model group and CSO treatment group. The treatment group was given CSO suspended in egg yolk intragastrically at a dose of $6 \mathrm{ml} \mathrm{CSO} /$ $\mathrm{kg} / \mathrm{d}$, and the model group was given vehicle control $(4 \%$ egg yolk). The tumor volume and the bodyweight of these mice were recorded daily. These mice were sacrificed after 21 days of continuous administration.

\section{Bioluminescent Imaging}

D-Luciferin (PerkinElmer, $150 \mu \mathrm{l}$ in PBS solution) was injected intraperitoneally into mice and imaged under IVIS Imaging System (Xenogen, USA), and Living Image Software (Xenogen) was used to quantify the photons/second(p/s) emitted.

\section{miRNA Sequence Analysis}

miRNA sequence analysis was performed to assess miRNA expression profiles in the paired tumor tissues from the model group and CSO administration group. The fold changes (FCs) in the expression of individual miRNA were calculated. Differentially expressed miRNAs with $\log 2|\mathrm{FC}|>2.0$ and $\mathrm{P}<$ 0.05 were considered to be significant.

\section{Lipid Metabolomics Analysis}

Blood samples collected from the mice were stored at $4^{\circ} \mathrm{C}$ for $1 \mathrm{~h}$, centrifuged at $4,000 \mathrm{rpm}\left(4^{\circ} \mathrm{C}, 15 \mathrm{~min}\right)$ by the centrifuge (Eppendorf, Hamburg, Germany), and the supernatant transferred into $1.5-\mathrm{ml}$ centrifuge tubes. Serum sample $(50 \mu \mathrm{l})$ was diluted with ultrapure Millipore water (1:1 ratio), mixed with chloroform/methanol $(2: 1$ ratio; $200 \mu \mathrm{l})$, vortexed, and centrifuged at $12,000 \mathrm{rpm}$ for $10 \mathrm{~min}$ at $4^{\circ} \mathrm{C}$. The lower organic phase was taken and repeat the operation once. Combine the two organic phases and dry them using vacuum centrifugation. Methanol/isopropanol (1:1 ratio; $200 \mu \mathrm{l})$ mixture was added to re-dissolve them, and centrifuge at $1,200 \mathrm{rpm}$ $\left(4^{\circ} \mathrm{C}, 10 \mathrm{~min}\right)$. The centrifuged sample $(150 \mu \mathrm{l})$ was taken to place in the inner tube of the liquid via 1 for the lipidomic test.

Chromatographic conditions: the instrument was HPLCLTQ-Orbitrap Elite (Thermo, Waltham, MA, USA); column was Thermo Hypersil Gold C18; mobile phase: Phase A (60\% water and $40 \%$ acetonitrile with $0.1 \%$ formic acid, $10 \mathrm{mmol}$ ammonium formate) and Phase B (10\% acetonitrile and 90\% isopropanol, containing $0.1 \%$ formic acid, $10 \mathrm{mmol}$ ammonium formate); elution procedure: $0 \sim 1.0 \mathrm{~min}, 70 \% \mathrm{~A} ; 1.0 \sim 2.0 \mathrm{~min}$, 70\% 55\% A; 2.0 12.0 min, 55\% 35\% A; $12.0 \sim 18.0 \mathrm{~min}, 35 \%$ $\sim 15 \% \mathrm{~A} ; 18.0 \sim 20.0 \mathrm{~min}, 15 \% \sim 0 \% \mathrm{~A} ; 20.0 \sim 25.0 \mathrm{~min}, 100 \% \mathrm{~B}$; 25.1 30.0 $\mathrm{min}, 100 \% \mathrm{~B}$; volume flow was $0.3 \mathrm{ml} / \mathrm{min}$, injection volume was $4 \mu \mathrm{l}$; and the column temperature was $40^{\circ} \mathrm{C}$.

Mass spectrometry conditions: ion source spray voltages were 3.5 and $3.2 \mathrm{kV}$ in the $\mathrm{ESI}^{+}$and $\mathrm{ESI}^{-}$modes, respectively. The temperature of capillary was set at $350^{\circ} \mathrm{C}$ with the sheath gas flow rate at 35 arb, aux gas flow at 15 arb, and sweep gas flow rate at 1 arb. The heater temperature was at $300^{\circ} \mathrm{C}$. The S-Lens RF level was set at 60 . The MS was operated at a resolving power of 6,000 in Full-scan mode (scan range: $100-1,000 \mathrm{~m} / \mathrm{z}$ ) and of 15,000 in the top 10 data-dependent MS mode (collision energy: 35) with dynamic exclusion setting of $10 \mathrm{~s}$.

\section{Immunohistochemistry (IHC)}

IHC assay was performed to assess the levels of Ki67 and S1PR1 proteins in tumor samples. The experimental procedure was minor modified as previously reported (You et al., 2018).

\section{Western Blotting Analysis}

Cells or tumor tissues were lysed by RIPA buffer containing phosphatase inhibitors and protease inhibitors. The concentration of protein of each group was measured by BCA assay. Proteins were separated by SDS-PAGE and transferred to the PVDF membrane. Membranes were incubated with special primary or secondary antibodies. After washing with TBST, the ECL kit was used for band detection.

\section{Transfection}

Transfection of miR-205-5p mimic and miRNA-NC into 4T1 cells was performed by RNAIMAX reagent according to the manufacturer's instructions.

\section{qRT-PCR Analysis}

The total RNA of tumor tissues and cells extracted using the Trizol reagent according to the manufacturer's instructions. miR-205-5p, miR-200a-3p, miR-429-3p, miR-200c-3p, miR200b-3p, and miR-141-3p were selected to validate the results of miRNA sequencing analysis.

\section{Dual-Luciferase Reporter Assay}

$4 \mathrm{~T} 1$ cells were co-transfected with miR-205 mimic and a luciferase reporter plasmid containing wild-type S1PR1 3'-UTR or the mutant S1PR1 3'-UTR, respectively. Following $48 \mathrm{~h}$ of transfection, luciferase activity was determined by the dualluciferase reporter assay system (Promega, Madison, WI, USA). Luciferase activity of renilla was normalized to that of firefly luciferase.

\section{Statistical Analysis}

All data were presented as mean \pm SD (standard deviation, SD) from three independent experiments. One-way analysis of variance was used among multiple groups of data by SPS 20.0 statistical software. $P<0.05$ was considered statistically significant.

\section{RESULTS}

\section{Main Compounds Identified in CSO by LC-MS}

LC-MS was performed to determine the main ingredients in CSO. The results showed six components including 1, 2Dilinoleoyl-3-palmitoyl-rac-glycerol, 1, 2- Dilinoleoyl-3-oleoylrac-glycerol, 1-palmitoyl-2-oleoyl-3-linoleoyl-rac-glycerol, 1, 2Dioleoyl-3-linoleoyl-rac-glycerol, 1, 2-Dioleoyl-3-palmitoyl-racglycerol and Glycerol trioleate existed in CSO. Identified compounds were listed in Table 1, and the results of MTT assay were presented in the supplementary materials. 
TABLE 1 | Measurement results of Coix Seed oil by LC-MS.

\begin{tabular}{|c|c|c|c|c|}
\hline NO. & $t_{R} / \min$ & Compounds & Molecular formula & Molecular mass \\
\hline 1 & 17.09 & 1,2-Dilinoleoyl-3-palmitoyl-rac-glycerol & $\mathrm{C} 55 \mathrm{H} 98 \mathrm{O} 6$ & 854 \\
\hline 2 & 17.27 & 1,2-Dilinoleoyl-3-oleoyl-rac-glycerol & $\mathrm{C} 57 \mathrm{H} 10006$ & 880 \\
\hline 3 & 17.71 & 1-palmitoyl-2-oleoyl-3-linoleoyl-rac-glycerol & $\mathrm{C} 55 \mathrm{H} 10006$ & 856 \\
\hline 4 & 17.85 & 1,2-Dioleoyl-3-linoleoyl-rac-glycerol & $\mathrm{C} 57 \mathrm{H} 102 \mathrm{O} 6$ & 882 \\
\hline 5 & 18.26 & 1,2-Dioleoyl-3-palmitoyl-rac-glycerol & $\mathrm{C} 55 \mathrm{H} 102 \mathrm{O} 6$ & 858 \\
\hline 6 & 18.40 & Glycerol trioleate & $\mathrm{C} 57 \mathrm{H} 104 \mathrm{O} 6$ & 884 \\
\hline
\end{tabular}

\section{CSO Inhibited Cell Viability, Colony Formation, and Tumor Growth of 4T1}

In order to evaluate the effect of CSO on tumor growth, a triplenegative $4 \mathrm{~T} 1$ cell-based murine orthotopic transplantation model was used. Compared to the model group, CSO significantly inhibited tumor growth in vivo. There was no significant difference in the bodyweight of mice between model and treatment groups, indicating that CSO did no observable toxicity (Figures 1A-D). In addition, IHC analysis showed that CSO significantly reduced the number of Ki67-positive cells in tumor tissues (Figure 1E). Before the termination of this experiment, we used the IVIS Imaging System to detect the bioluminescence signal of the tumor in these two groups of mice. The luminescence signal of the tumors in the CSO administration group was weaker than that of the model group (Figures 1F, G). A nude mouse model based on the triple-negative MDA-MB-231 cell was also used and detailed data has been supplemented in Figure S3. These results suggested that CSO can effectively inhibit mammary carcinomabased tumor growth in vivo.

To further investigate the anticancer potential of CSO in vitro, we tested CSO on the viability of $4 \mathrm{~T} 1$ cells and colony formation. The results showed CSO decreased 4T1 cell viability and inhibited $4 \mathrm{~T} 1$ cell colony formation in a concentration-dependent manner compared to the untreated cells (Figures 1H, I).

These results suggested CSO effectively inhibited 4T1 proliferation, colony formation in vitro, and tumor growth in vivo.

\section{CSO Strongly Elevated miR-205-5p in Tumor Tissues}

miRNA sequencing analysis was carried out to assess the alteration of miRNAs in tumor tissues after CSO administration. The result revealed the top 10 up-regulated miRNA included miR-205-5p, miR-200a-3p, miR-429-3p, miR-200c-3p, miR-200b-3p, miR-2033p, miR-133b-3p, miR-141-3p, miR-206-3p, and miR-133a-3p and the only down-regulated miR-669f-3p (Table 2). Further qRT-PCR analysis of tumor tissue samples confirmed the sequencing results. Among the identified microRNAs, miR-205$5 \mathrm{p}$ was most upregulated under CSO treatment among these key miRNAs (Figures 2A, B). These results indicated that CSO could strongly increase the level of miR-205-5p in tumor tissues.

\section{CSO Increased Sphingomyelin Metabolism (SM) in the Serum}

Due to the oily nature of CSO, we speculated that the tumorsuppressive effect of CSO might be related to lipid metabolism. To test this hypothesis, we performed lipid metabolomics assay and found that compared with normal mouse serum, the level of sphingomyelin in the serum of the 4T1 mammary carcinoma allograft mice decreased significantly, and this phenomenon had a significant callback trend after CSO administration (Figure 3). This result demonstrated that the tumor-suppressive effect of CSO was related to the increase of SM.

\section{miR-205-5p Regulated SM Partly via Targeting S1PR1 to Suppress Cell Proliferation}

Since miR-205-5p might act as a tumor suppressor miRNA in the development of $\mathrm{BC}$, we transfected miR-205 mimic or miRNA NC into $4 \mathrm{~T} 1$ cells to compare cell proliferation between these groups.

MTT assay result showed that over-expressed miR-205 decreased the ability of cell growth. The expressions of CyclinD1 and p27, the critical mediators in the cell cycle, were regulated by miR-205 after 72 -h transfection. These results showed miR-205 could block the cell cycle and proliferation (Figures 4A, B).

In order to investigate the correlation between miR-205-5p and $\mathrm{SM}$, we performed the lipidomic analysis on miR-205-5p overexpressed 4T1 cells. Overexpressed miR-205-5p in 4T1 partially increased SM compared to the NC group. Here, we only listed a part of the SM with a higher-fold increase, and these results indicated that miR-205-5p could increase SM accumulation (Figure 4C).

We performed bioinformatics analysis to predict targets of miR205 and found a potential target S1PR1 related to SM in Target RNA Mouse 7.1 (Lewis et al., 2005). Therefore, we examined the protein levels of S1PR1 after miR-205-5p overexpression by western blotting. The up-regulation of miR-205-5p led to the decreased protein expression of S1PR1 compared to the NC group (Figure 4D). This result suggested that miR-205-5p could negatively regulate the expression of S1PR1.

In order to validate whether S1PR1 was a target of miR-205, a luciferase reporter assay was carried out. Luciferase activity was reduced in 4T1 cells co-transfected with the wild-type 3'UTR of S1PR1 and miR-205 mimic. However, luciferase activity remained unchanged in the 4T1 cells co-transfected with mutant S1PR1 3'UTR and miR-205 mimic. These data indicated that S1PR1 might be a novel target of miR-205 (Figure 4E).

Furthermore, to evaluate the roles of S1PR1 on cell proliferation, we performed MTT assay. After transfection for $72 \mathrm{~h}$, the gene expression of S1PR1 was decreased in siRNAS1PR1 treated cells compared to the siRNA-NC group (Figure 4F). And knockdown of S1PR1 significantly inhibited 4T1 cell proliferation (Figure 4G). 


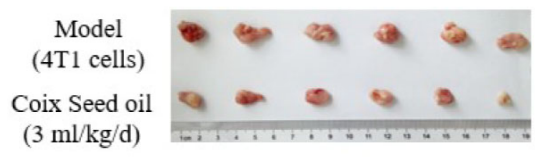

C

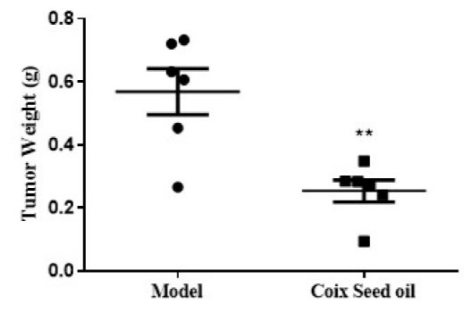

E
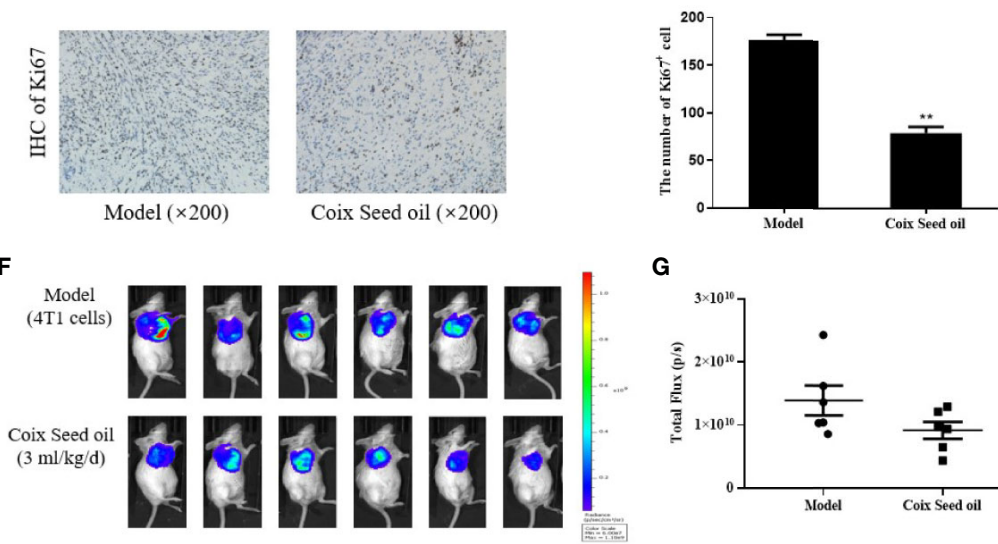

G
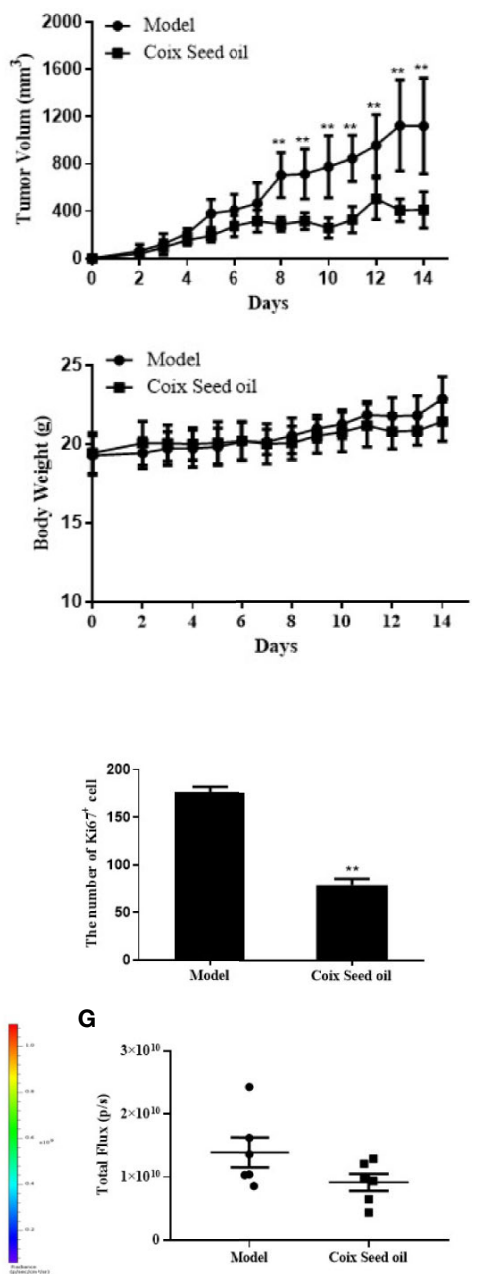$$
\text { . }
$$

H

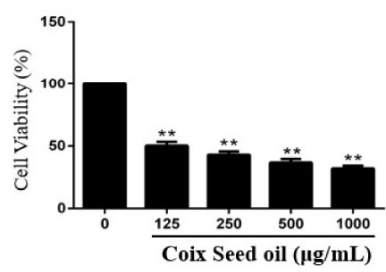

I

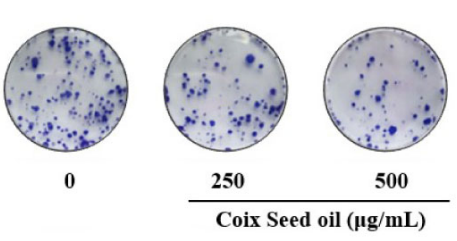


TABLE 2 | Micro RNA sequencing results.

\section{Micro RNA}

mmu-miR-205-5p

mmu-miR-200a-3p

mmu-miR-429-3p

mmu-miR-200c-3p

mmu-miR-200b-3p

mmu-miR-203-3p

mmu-miR-133b-3p

mmu-miR-141-3p

mmu-miR-206-3p

mmu-miR-133a-3p

mmu-miR-669f-3p

${ }^{* *} P<0.01$ vs. model.

S1PR1 exerts its functions via downstream signal pathways including STAT3. In addition, STAT3 phosphorylation in 4T1 cells was significantly reduced after miR-205-5p overexpression (Figure 4H). This result showed the STAT3 signaling pathway was involved in miR-205-S1PR1-led effects on cell proliferation.

\section{CSO Reduced S1PR1, CyclinD1 Expressions, and Increased p27 Expression}

In order to determine whether CSO can regulate S1PR1, CyclinD1, and p27 expression due to the elevated miR-205, Western blot, qPCR, and IHC were performed to detect the changes of S1PR1 upon CSO treatment. CSO significantly downregulated S1PR1 and Cyclin D1 expression and upregulated the expression level of p27 in tumor tissues and cells (Figures 5A, B, D). Besides, IHC results showed that CSO significantly reduced the expression level of S1PR1 in tumor tissue sections (Figure 5C). These results suggested that CSO regulated cell cycle and inhibited tumor growth through the miR-205/S1PR1 axis.

\section{Proliferation-Related Pathways Were Involved in CSO-Mediated Effects}

Accumulating studies have confirmed that STAT3 is a transcription regulator of S1PR1 gene expression. Therefore, we

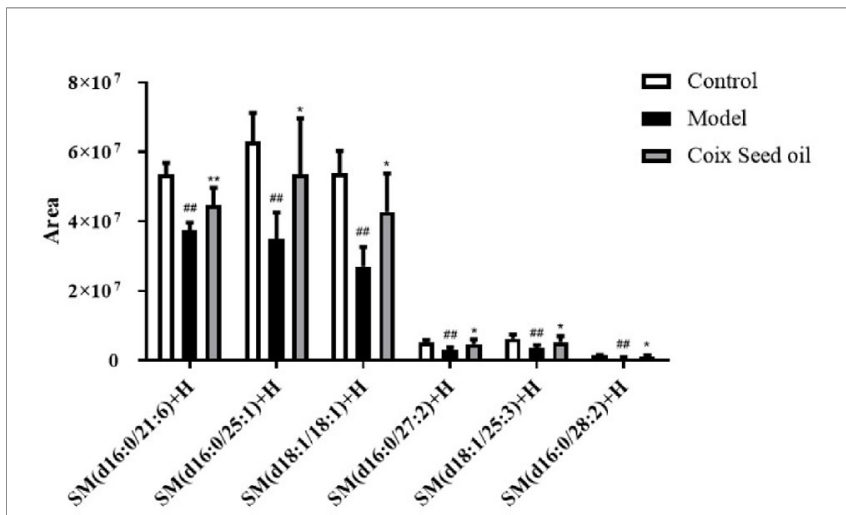

FIGURE 3 | Effect of CSO on the levels of serum sphingomyelin in the 4T1 mammary carcinoma allograft mice. Compared with the control group, the content of sphingomyelin in the serum of the 4T1 allograft mice changed. The sphingomyelin increased in the CSO treatment group(3 ml/kg for 14 days). ${ }^{\# \#} p<0.01$ vs. control and ${ }^{*} p<0.05,{ }^{* *} p<0.01$ vs. model.

tested whether CSO can inhibit the phosphorylation of STAT3 in TNBC by western blotting. Treatment of CSO significantly downregulated phosphorylation of STAT3 in vivo and in vitro (Figures 6A, B). These data showed that the inactivation of the STAT3 pathway contributed to the downregulation of S1PR1 of the CSOled effect. We also evaluated its effect on the activation of other proliferation-related pathways such as MAPK and AKT. The treatment with CSO significantly inhibited the phosphorylation of ERK, JNK, p38, and AKT without no effect on the total of ERK, JNK, p38, and AKT in vivo and in vitro (Figures 6C, D). Our results suggest that three important proliferation-related pathways including STAT3, MAPK, and AKT were involved in CSOled effects.

\section{DISCUSSION}

CSO processes antitumor activities, especially on lung and liver cancers, according to the increasing experimental and clinical
A

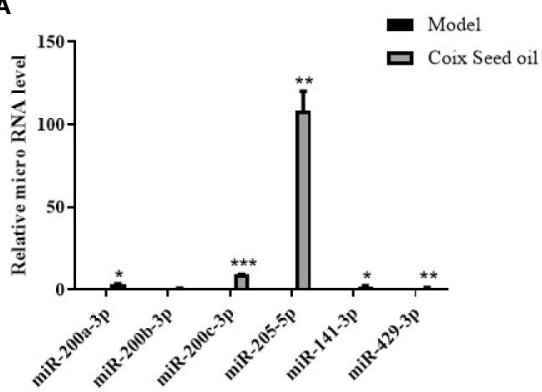

B

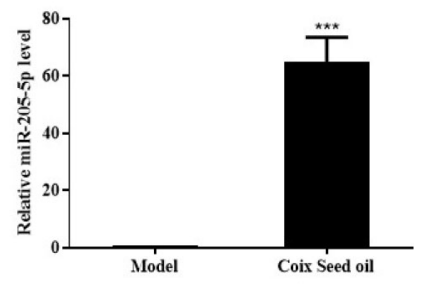

FIGURE 2 | Effect of CSO on the level of miR-205-5p in the tumor tissues of the 4T1 mammary carcinoma allograft mice. (A) qRT-PCR analysis was used to detect the expression of miRNAs. Compared with the model group, the levels of miR-200a-3p, miR-200b-3p, miR-200c-3p, miR-205-5p, miR-141-3p, and miR-429-3p increased in the CSO treated group ( $3 \mathrm{ml} / \mathrm{kg}, 14$ days). (B) The expression of miR-205-5p was detected from mixed tumor tissues in two groups by qRT-PCR. Compared with the model group, the level of miR-205-5p from the mixture of tumor tissues increased in the individuals CSO treated $\left(3 \mathrm{ml} / \mathrm{kg}, 14\right.$ days). ${ }^{\star} P<0.05$, ${ }^{* *} P<0.01,{ }^{* *} P<0.001$ vs. model. 
A

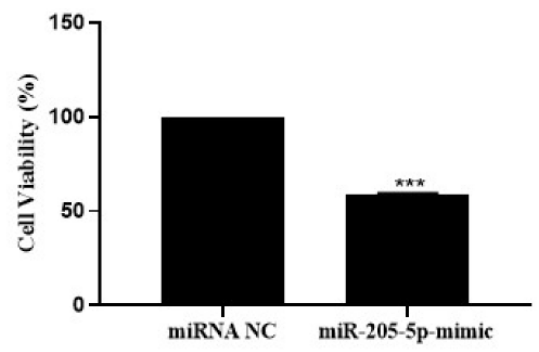

B

NC miR-205-5p mimics

Cyclin D1

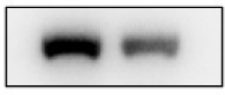

p27

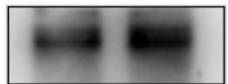

GAPDH

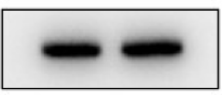

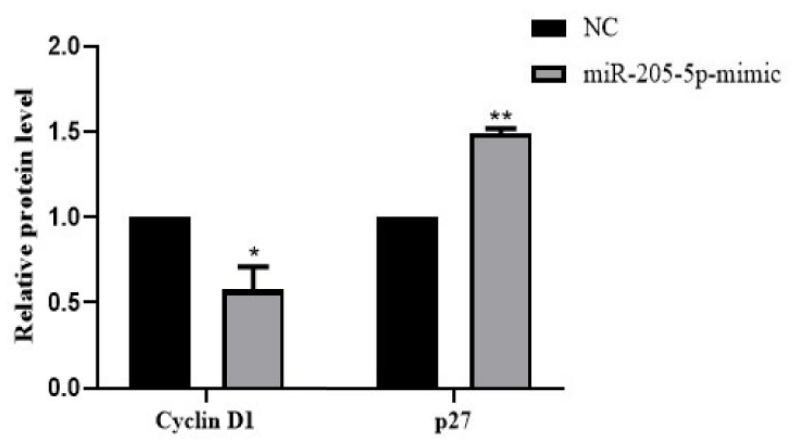

C

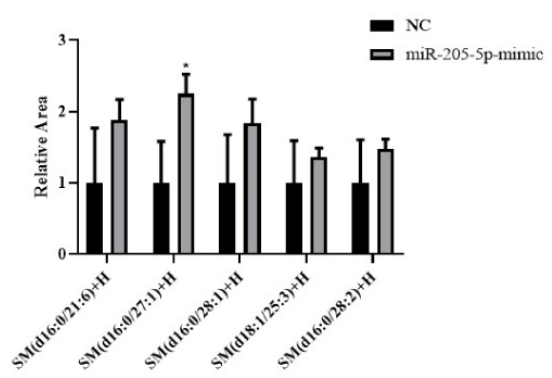

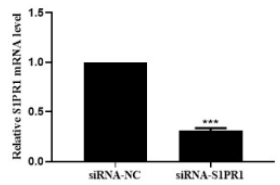

H

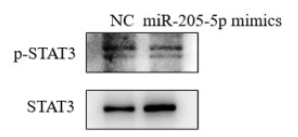

G

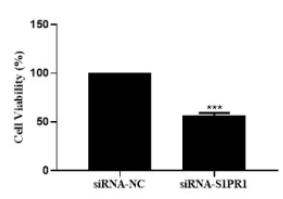

E
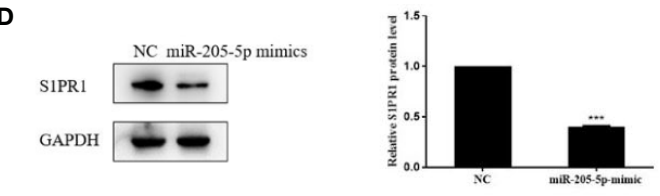

WT SIPRI 3'-UTR AGUUUCCUGAAGGAA... miR-205 CCACCUUACUUCCU... MUT SIPRI 3'-UTR AGUUUCCACUUCCAA...

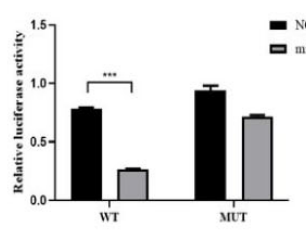
- $\mathrm{NC}$
aiR-20s.sp-mimic miR/2005.5p-mimic

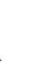

$$
\text { (1) }
$$


A

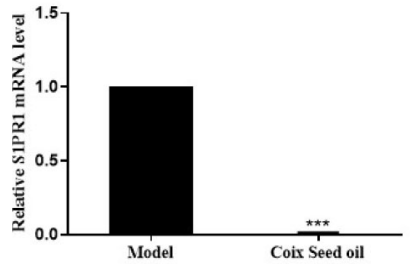

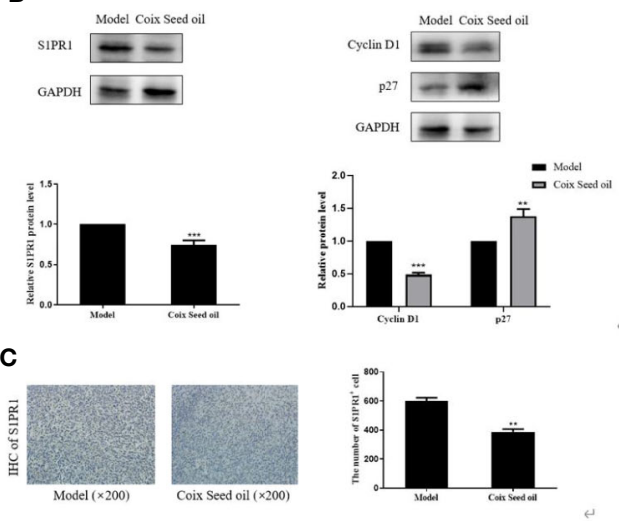
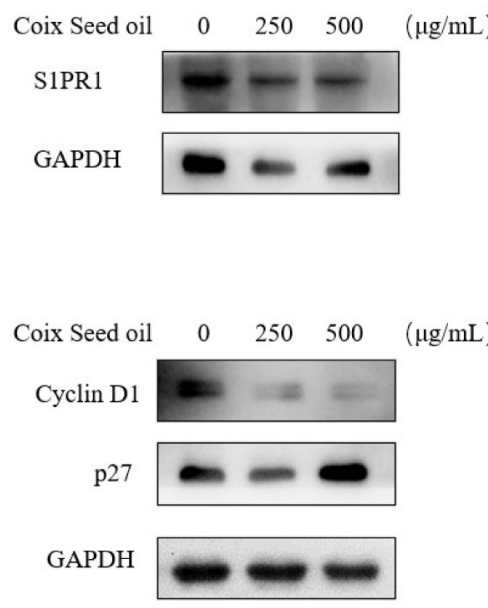

$\mu \mathrm{g} / \mathrm{mL})$
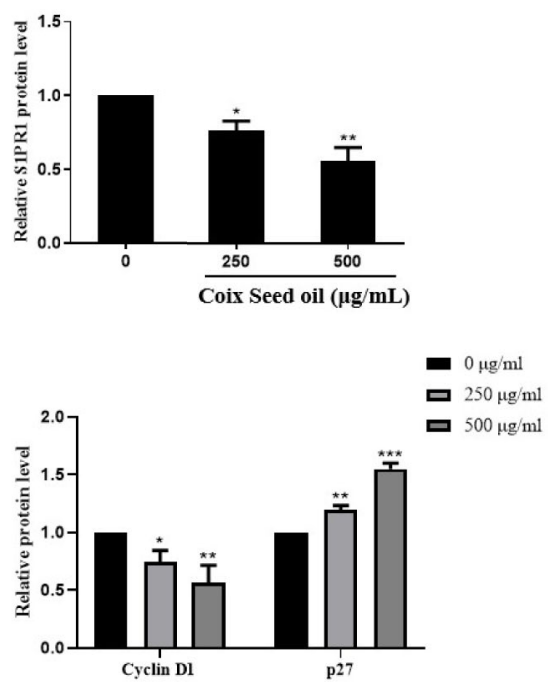

FIGURE 5 | Effects of CSO on S1PR1, Cyclin D1, and p27 expressions in tumor tissues and in cultured cells. (A) The gene expression of the S1PR1 in tumor tissues. (B) The protein expressions of S1PR1, Cyclin D1, and p27 in tumor tissues. (C) IHC analysis of S1PR1 of tumor sections. (D) The protein expressions of S1PR1, Cyclin D1, and p27 in 4 T1 cells. ${ }^{*} p<0.05,{ }^{* *} p<0.01{ }^{* * *} p<0.001$ vs. untreated.

evidence. However, the effect of CSO on TNBC and underlying mechanisms related to miRNA alteration remains to be explored. MicroRNA (miRNA) is a type of cellular non-coding small single-stranded RNA that can bind to the complementary sequence on the 3'UTR of specific mRNA, which results in the degradation or unstable condition of the target gene (Croce and Calin, 2005; Kian et al., 2018). In breast cancer, altered expression of specific miRNA may promote or suppress oncogenesis. Among these miRNAs, miR-205-5p abnormally expressed in a variety of tumors. Previous studies showed that miR-205-5p could function as a tumor suppressor in breast cancer (Zhang and Fan, 2015; Wang et al., 2019), prostate cancer (Tucci et al., 2012; Li and Li, 2018), kidney cancer (Chen et al., 2014), and melanoma (Chen et al., 2019), while act as an oncogene in the development of lung cancer (Jiang et al., 2017) and ovarian cancer (Wei et al., 2017). A large amount of evidence proved that the increased expression of miR-205-5p in tumor cells could significantly inhibit the proliferation and metastasis of breast cancer cells, especially TNBC cells (Xiao et al., 2019). Our data showed CSO blocked cell proliferation and tumor growth of TNBC 4T1 cells with an increase of miR-205 by miRNA-sequencing analysis (Figures 1, 2).

The main ingredients in CSO are fatty acids such as palmitic, stearic, oleic, and linoleic acids (Numata et al., 1994), which contribute to its anticancer activity. But the link between CSO and lipid metabolomics in TNBC is still unclear. Recent studies have demonstrated that Altered and reprogrammed sphingomyelin metabolism is strictly related to the progression of numerous cancers and has been identified as a hallmark of cancer aggressiveness. Sphingomyelin and its metabolites take participate in cellular signaling processes including cell growth, differentiation, senescence, and programmed cell death (Ogretmen and Hannun, 2004). Accumulation of sphingomyelins was significantly linked with better prognosis and disease-free survival outcomes in TNBC patients (Purwaha et al., 2018). The result of lipid metabolomics analysis showed CSO administration caused the SM accumulation in tumorbearing mice (Figure 3). 
A
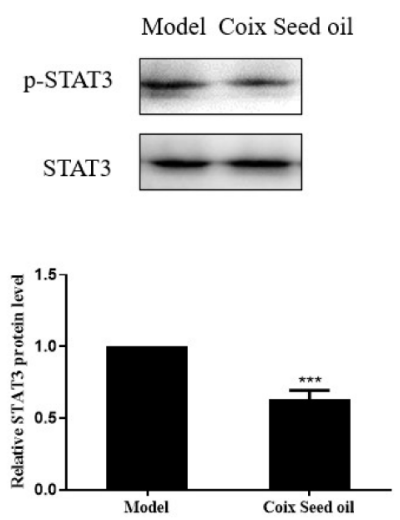

C

D

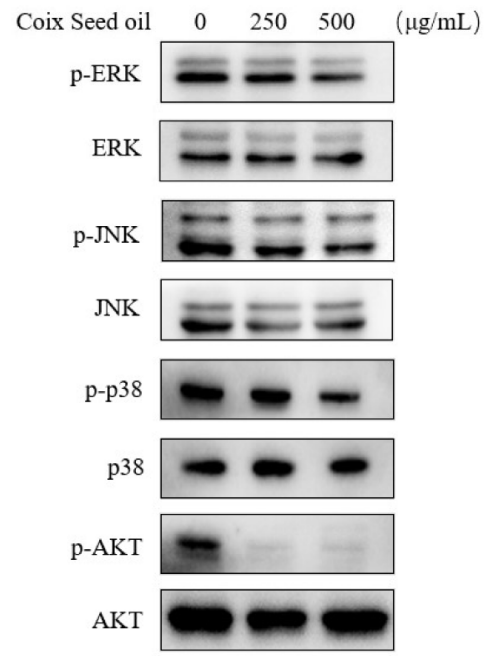

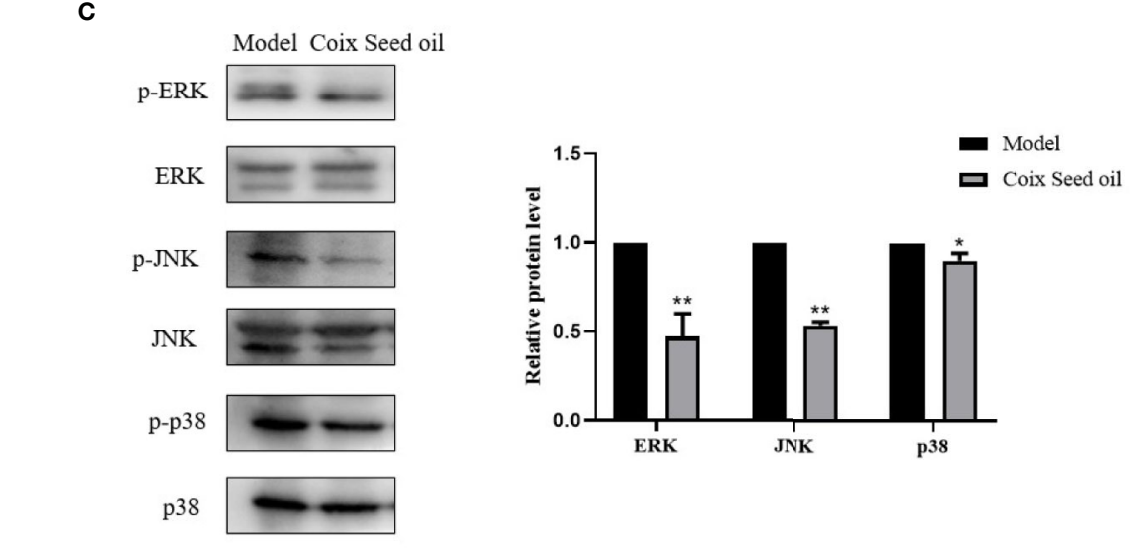
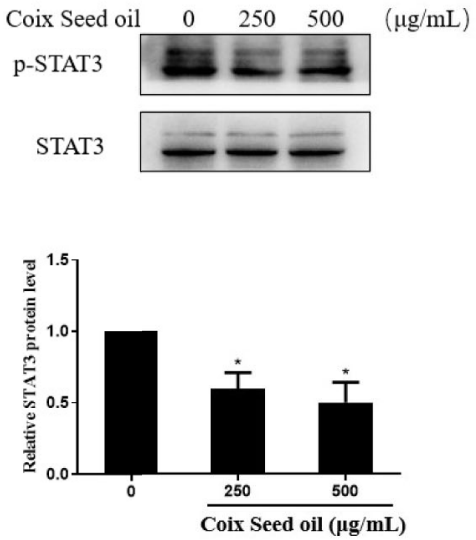

| Effects of CSO on proliferation-related pathways in tumor tissues and cells. (A) The level of STAT3 phosphorylation in tumor tissues. (B) The level of STAT3 phosphorylation in 4T1 cells. (C) Phosphorylation levels of ERK, JNK, p38, AKT in tumor tissue. (D) Phosphorylation levels of ERK, JNK, p38, and AKT in 4T1 cells. ${ }^{*} p<0.05,{ }^{* *} p<0.01{ }^{* *} p<0.001$ vs. untreated. 
miR-205 is a well-established miRNA in breast cancer. Nevertheless, its function in SM regulation has no previously been reported. Sphingosine-1-phosphate (S1P) and its receptors (sphingosine 1 phosphate receptors, S1PRs) in sphingomyelin metabolism are important signaling effectors involved in regulating cell growth, differentiation, senescence, and death (Pyne and Pyne, 2011; Adada et al., 2016; Ogretmen, 2018). The function of S1P depends on its receptors named S1PRs. Among S1P receptors, S1PR1 is implicated in tumorigenesis and progression (Waters et al., 2003; Brocklyn, 2010; Nagahashi et al., 2014; Weichand et al., 2017; Ogretmen, 2018; Rostami et al., 2019). S1PR1 increased its expression in TNBC. FTY720 reduces tumor growth, metastasis, angiogenesis, and enhances drugsensitivity of TNBC as an antagonist of S1PR1 (Nagahashi et al., 2018). FTY720 targets the SphK1/S1P/S1PR1 axis including induction of polyubiquitylation and degradation of S1PR1 to regulate sphingolipid metabolism (Graler and Goetzl, 2004). Therefore, S1PR1 emerged as a potential therapeutic target for TNBC.

Our findings confirmed the association between miR-205 and S1PR1 in TNBC cells that S1PR1 is a direct target of miR-205. miR-205 directly targeted S1PR1 to regulate SM and consequent cell proliferation (Figure 4) and tumor development.

Cyclin D1 is a tumor-associated protooncogene recognized in recent years and overexpression in various tumor cells (Musgrove et al., 2011). The p27 protein is a tumor suppressor gene discovered in recent years. It blocks the cell transition from the quiescent phase to the proliferative phase in the cell cycle, thereby inhibiting cell proliferation and is a negative regulatory factor of the cell cycle (Chu et al., 2008).

CSO treatment repressed the expression of S1PR1 in cells and tumor tissues accompanied by the downregulated expression of cyclinD1 and upregulated expression of p27 (Figure 5).

The S1PR1 signaling can regulate the survival of breast cancer cells, which can increase the survival of breast cancer cells by downregulating the pro-apoptotic protein Bim and upregulating the anti-apoptotic protein Mcl-1, respectively, in ERK and PKCdependent manner (Rutherford et al., 2013). In addition, one of the main functions of the activation of the S1PR1 signaling pathway is the continuous activation of STAT3 in the form of phosphorylation, leading to overexpression of NF- $\mathrm{KB}$ and IL-6, which promotes tumor development (Liang et al., 2013). Studies have shown that STAT3 is a transcription factor for the S1PR1 gene, and enhanced expression of S1PR1 can continuously activate STAT3 and upregulate the expression of the IL- 6 gene, thereby accelerating tumor growth and metastasis (Liu et al., 2012; Liang et al., 2013). Similarly, studies indicate that S1PR1 can interact with JAK2, resulting in increased STAT3 phosphorylation, while activated STAT3 can, in turn, upregulate the expression of S1PR1 transcript levels, thus forming a feedforward cycle (Jin et al., 2016). Thus, targeted inhibition of the S1PR1/STAT3 can inhibit tumor proliferation and growth in a STAT3-dependent manner. Experimental evidence implicated S1PR1 coupled to Gi and persistently triggered STAT3, mitogen-activated protein kinase (MAPK) and AKT signaling pathways, to promote many types of tumor growth and metastasis including breast cancer (Lee et al., 2010; Deng et al., 2012; Paik et al., 2014; Go et al., 2015).

Our finding showed miR205-S1PR1 axis regulated activation of the STAT3 signal pathway (Figures 6A, B) as well as MAPK and AKT pathways (Figures 6C, D).

\section{CONCLUSION}

CSO exerted an anti-TNBC effect via the miR-205/S1PR1 axis to regulate sphingomyelin metabolism, and the downstream STAT3/MAPK/AKT signal pathways were partly involved. CSO may serve as a potential drug for the treatment of TNBC.

\section{DATA AVAILABILITY STATEMENT}

All datasets generated for this study are included in the article/ Supplementary Material.

\section{ETHICS STATEMENT}

The protocol in the animal experiment of this study was reviewed and approved by the Institutional Animal Care and Use Committee of Shanghai University of Traditional Chinese Medicine.

\section{AUTHOR CONTRIBUTIONS}

TF performed the cell and animal experiments and wrote the manuscript. Y-XJ established the animal model and following CSO administration, and LC performed lipid metabolomics analysis. LH analyzed the data, X-HT performed LC-MS analysis of CSO. Y-DZ and DN modified the manuscript. D-DZ designed this study. All authors contributed to the article and approved the submitted version.

\section{FUNDING}

This work was supported by the National Natural Science Foundation of China (81773946, 81573673, and 81001666); Innovation project for undergraduates of shanghai university of Traditional Chinese Medicine (2019SHUTCM168).

\section{SUPPLEMENTARY MATERIAL}

The Supplementary Material for this article can be found online at: https://www.frontiersin.org/articles/10.3389/fphar.2020.529962/ full\#supplementary-material 


\section{REFERENCES}

Adada, M., Luberto, C., and Canals, D. (2016). Inhibitors of the sphingomyelin cycle: Sphingomyelin synthases and sphingomyelinases. Chem. Phys. Lipids 197, 45-59. doi: 10.1016/j.chemphyslip.2015.07.008

Bray, F., Ferlay, J., Soerjomataram, I., Siegel, R. L., Torre, L. A., and Jemal, A. (2018). Global cancer statistics 2018: GLOBOCAN estimates of incidence and mortality worldwide for 36 cancers in 185 countries. CA Cancer J. Clin. 68 (6), 394-424. doi: $10.3322 /$ caac. 21492

Brocklyn, J. R. (2010). Regulation of cancer cell migration and invasion by sphingosine-1-phosphate. World J. Biol. Chem. 1 (10), 307-312. doi: 10.4331 /wjbc.v1.i10.307

Chen, Z., Tang, Z. Y., He, Y., Liu, L. F., Li, D. J., and Chen, X. (2014). miRNA-205 is a candidate tumor suppressor that targets ZEB2 in renal cell carcinoma. Oncol. Res. Treat 37 (11), 658-664. doi: 10.1159/000368792

Chen, Y., Cao, K., Li, J., Wang, A., Sun, L., Tang, J., et al. (2019). Overexpression of long non-coding RNA NORAD promotes invasion and migration in malignant melanoma via regulating the MIR-205-EGLN2 pathway. Cancer Med. 8 (4), 1744-1754. doi: 10.1002/cam4.2046

Chu, I. M., Hengst, L., and Slingerland, J. M. (2008). The Cdk inhibitor p27 in human cancer: prognostic potential and relevance to anticancer therapy. Nat. Rev. Cancer 8 (4), 253-267. doi: 10.1038/nrc2347

Croce, C. M., and Calin, G. A. (2005). miRNAs, cancer, and stem cell division. Cell 122 (1), 6-7. doi: 10.1016/j.cell.2005.06.036

Deng, J., Liu, Y., Lee, H., Herrmann, A., Zhang, W., Zhang, C., et al. (2012). S1PR1-STAT3 signaling is crucial for myeloid cell colonization at future metastatic sites. Cancer Cell 21 (5), 642-654. doi: 10.1016/j.ccr.2012.03.039

Duan, G. C. (2018). The Effects of Combination of Coix Seed Extract and Cisplatin on TAM and Expression of HIF-1alpha in Vivo in Lewis Lung Carcinoma. Iran J. Public Health 47 (6), 838-843.

Go, H., Kim, P. J., Jeon, Y. K., Cho, Y. M., Kim, K., Park, B. H., et al. (2015). Sphingosine-1-phosphate receptor 1 (S1PR1) expression in non-muscle invasive urothelial carcinoma: Association with poor clinical outcome and potential therapeutic target. Eur. J. Cancer 51 (14), 1937-1945. doi: 10.1016/ j.ejca.2015.07.021

Graler, M. H., and Goetzl, E. J. (2004). The immunosuppressant FTY720 downregulates sphingosine 1-phosphate G-protein-coupled receptors. FASEB J. 18 (3), 551-553. doi: 10.1096/fj.03-0910fje

Guo, H. Y., Cai, Y., Yang, X. M., Wang, Z. H., Wang, J. L., Zhao, X. M., et al. (2008). Randomized phase II trial on mitomycin-C/cisplatin +/- KLT in heavily pretreated advanced breast cancer. Am. J. Chin. Med. 36 (4), 665674. doi: $10.1142 / S 0192415 X 08006132$

Jiang, M., Zhong, T., Zhang, W., Xiao, Z., Hu, G., Zhou, H., et al. (2017). Reduced expression of miR2055p promotes apoptosis and inhibits proliferation and invasion in lung cancer A549 cells by upregulation of ZEB2 and downregulation of erbB3. Mol. Med. Rep. 15 (5), 3231-3238. doi: 10.3892/ mmr.2017.6398

Jin, L., Liu, W. R., Tian, M. X., Fan, J., and Shi, Y. H. (2016). The SphKs/S1P/ S1PR1 axis in immunity and cancer: more ore to be mined. World J. Surg. Oncol. 14, 131. doi: 10.1186/s12957-016-0884-7

Kian, R., Moradi, S., and Ghorbian, S. (2018). Role of components of microRNA machinery in carcinogenesis. Exp. Oncol. 40 (1), 2-9. doi: 10.31768/23128852.2018.40(1):2-9

Lee, H., Deng, J., Kujawski, M., Yang, C., Liu, Y., Herrmann, A., et al. (2010). STAT3-induced S1PR1 expression is crucial for persistent STAT3 activation in tumors. Nat. Med. 16 (12), 1421-1428. doi: 10.1038/nm.2250

Lewis, B. P., Burge, C. B., and Bartel, D. P. (2005). Conserved seed pairing, often flanked by adenosines, indicates that thousands of human genes are microRNA targets. Cell 120 (1), 15-20. doi: 10.1016/i.cell.2004.12.035

Li, L., and Li, S. (2018). miR-205-5p inhibits cell migration and invasion in prostatic carcinoma by targeting ZEB1. Oncol. Lett. 16 (2), 1715-1721. doi: 10.3892/ol.2018.8862

Liang, J., Nagahashi, M., Kim, E. Y., Harikumar, K. B., Yamada, A., Huang, W. C., et al. (2013). Sphingosine-1-phosphate links persistent STAT3 activation, chronic intestinal inflammation, and development of colitis-associated cancer. Cancer Cell 23 (1), 107-120. doi: 10.1016/j.ccr.2012.11.013

Liu, Y., Deng, J., Wang, L., Lee, H., Armstrong, B., Scuto, A., et al. (2012). S1PR1 is an effective target to block STAT3 signaling in activated B cell-like diffuse large
B-cell lymphoma. Blood 120 (7), 1458-1465. doi: 10.1182/blood-2011-12399030

Liu, Y., Zhang, W., Wang, X. J., and Liu, S. (2014). Antitumor effect of Kanglaite (R) injection in human pancreatic cancer xenografts. BMC Complement. Altern. Med. 14, 228. doi: 10.1186/1472-6882-14-228

Liu, H., Li, L., Zou, J., Zhou, T., Wang, B., Sun, H., et al. (2019). Coix seed oil ameliorates cancer cachexia by counteracting muscle loss and fat lipolysis. BMC Complement. Altern. Med. 19 (1), 267. doi: 10.1186/s12906-019-2684-4

Lu, Y., Li, C. S., and Dong, Q. (2008). Chinese herb related molecules of cancercell-apoptosis: a minireview of progress between Kanglaite injection and related genes. J. Exp. Clin. Cancer Res. 27:31. doi: 10.1186/1756-9966-27-31

Miller, K. D., Nogueira, L., Mariotto, A. B., Rowland, J. H., Yabroff, K. R., Alfano, C. M., et al. (2019). Cancer treatment and survivorship statistic. CA Cancer J. Clin. 69 (5), 363-385. doi: 10.3322/caac.21565

Musgrove, E. A., Caldon, C. E., Barraclough, J., Stone, A., and Sutherland, R. L. (2011). Cyclin D as a therapeutic target in cancer. Nat. Rev. Cancer 11 (8), 558572. doi: $10.1038 / \mathrm{nrc} 3090$

Nagahashi, M., Hait, N. C., Maceyka, M., Avni, D., Takabe, K., Milstien, S., et al. (2014). Sphingosine-1-phosphate in chronic intestinal inflammation and cancer. Adv. Biol. Regul. 54, 112-120. doi: 10.1016/j.jbior.2013.10.001

Nagahashi, M., Yamada, A., Katsuta, E., Aoyagi, T., Huang, W. C., Terracina, K. P., et al. (2018). Targeting the SphK1/S1P/S1PR1 Axis That Links Obesity, Chronic Inflammation, and Breast Cancer Metastasis. Cancer Res. 78 (7), 1713-1725. doi: 10.1158/0008-5472.CAN-17-1423

Numata, M., Yamamoto, A., Moribayashi, A., and Yamada, H. (1994). Antitumor components isolated from the Chinese herbal medicine Coix lachryma-jobi. Planta Med. 60 (4), 356-359. doi: 10.1055/s-2006-959500

Ogretmen, B., and Hannun, Y. A. (2004). Biologically active sphingolipids in cancer pathogenesis and treatment. Nat. Rev. Cancer 4 (8), 604-616. doi: $10.1038 / \mathrm{nrc1} 141$

Ogretmen, B. (2018). Sphingolipid metabolism in cancer signalling and therapy. Nat. Rev. Cancer 18 (1), 33-50. doi: 10.1038/nrc.2017.96

Paik, J. H., Nam, S. J., Kim, T. M., Heo, D. S., Kim, C. W., and Jeon, Y. K. (2014). Overexpression of sphingosine-1-phosphate receptor 1 and phospho-signal transducer and activator of transcription 3 is associated with poor prognosis in rituximab-treated diffuse large B-cell lymphomas. BMC Cancer 14:911. doi: 10.1186/1471-2407-14-911

Purwaha, P., Gu, F., Piyarathna, D., Rajendiran, T., Ravindran, A., Omilian, A. R., et al. (2018). Unbiased Lipidomic Profiling of Triple-Negative Breast Cancer Tissues Reveals the Association of Sphingomyelin Levels with Patient DiseaseFree Survival. Metabolites 8 (3), 41. doi: 10.3390/metabo8030041

Pyne, S., and Pyne, N. J. (2011). Translational aspects of sphingosine 1-phosphate biology. Trends Mol. Med. 17 (8), 463-472. doi: 10.1016/j.molmed.2011.03.002

Rostami, N., Nikkhoo, A., Ajjoolabady, A., Azizi, G., Hojjat-Farsangi, M., Ghalamfarsa, G., et al. (2019). S1PR1 as a Novel Promising Therapeutic Target in Cancer Therapy. Mol. Diagn. Ther. 23 (4), 467-487. doi: 10.1007/ s40291-019-00401-5

Rutherford, C., Childs, S., Ohotski, J., McGlynn, L., Riddick, M., MacFarlane, S., et al. (2013). Regulation of cell survival by sphingosine-1-phosphate receptor S1P1 via reciprocal ERK-dependent suppression of Bim and PI-3-kinase/ protein kinase C-mediated upregulation of Mcl-1. Cell Death Dis. 4, e927. doi: $10.1038 /$ cddis. 2013.455

Schwartzberg, L. S., Arena, F. P., Bienvenu, B. J., Kaplan, E. H., Camacho, L. H., Campos, L. T., et al. (2017). A Randomized, Open-Label, Safety and Exploratory Efficacy Study of Kanglaite Injection (KLTi) plus Gemcitabine versus Gemcitabine in Patients with Advanced Pancreatic Cancer. J. Cancer 8 (10), 1872-1883. doi: 10.7150/jca.15407

Tucci, P., Agostini, M., Grespi, F., Markert, E. K., Terrinoni, A., Vousden, K. H., et al. (2012). Loss of p63 and its microRNA-205 target results in enhanced cell migration and metastasis in prostate cancer. Proc. Natl. Acad. Sci. U. S. A. 109 (38), 15312-15317. doi: 10.1073/pnas.1110977109

Wang, L., Kang, F. B., Wang, J., Yang, C., and He, D. W. (2019). Downregulation of miR-205 contributes to epithelial-mesenchymal transition and invasion in triple-negative breast cancer by targeting HMGB1-RAGE signaling pathway. Anticancer Drugs 30 (3), 225-232. doi: 10.1097/CAD.0000000000000705

Waters, C., Sambi, B., Kong, K. C., Thompson, D., Pitson, S. M., Pyne, S., et al. (2003). Sphingosine 1-phosphate and platelet-derived growth factor (PDGF) act via PDGF beta receptor-sphingosine 1-phosphate receptor complexes in 
airway smooth muscle cells. J. Biol. Chem. 278 (8), 6282-6290. doi: 10.1074/ jbc.M208560200

Wei, J., Zhang, L., Li, J., Zhu, S., Tai, M., Mason, C. W., et al. (2017). MicroRNA205 promotes cell invasion by repressing TCF21 in human ovarian cancer. J. Ovarian Res. 10 (1), 33. doi: 10.1186/s13048-017-0328-1

Weichand, B., Popp, R., Dziumbla, S., Mora, J., Strack, E., Elwakeel, E., et al. (2017). S1PR1 on tumor-associated macrophages promotes lymphangiogenesis and metastasis via NLRP3/IL-1beta. J. Exp. Med. 214 (9), 2695-2713. doi: 10.1084/jem.20160392

Wu, Y., Zhang, J., Hong, Y., and Wang, X. (2018). Effects of Kanglaite Injection on Serum miRNA-21 in Patients with Advanced Lung Cancer. Med. Sci. Monit. 24, 2901-2906. doi: 10.12659/MSM.909719

Xiao, Y., Humphries, B., Yang, C., and Wang, Z. (2019). MiR-205 Dysregulations in Breast Cancer: The Complexity and Opportunities. Noncoding RNA 5 (4), 53. doi: $10.3390 /$ ncrna5040053

Yang, C., Hou, A., Yu, C., Dai, L., Wang, W., Zhang, K., et al. (2018). Kanglaite reverses multidrug resistance of HCC by inducing apoptosis and cell cycle arrest via PI3K/ AKT pathway. Onco. Targets Ther. 11, 983-996. doi: 10.2147/OTT.S153814

You, X., Wang, Y., Wu, J., Liu, Q., Chen, D., Tang, D., et al. (2018). Galectin-1 Promotes Metastasis in Gastric Cancer Through a Sphingosine-1-Phosphate Receptor 1-Dependent Mechanism. Cell. Physiol. Biochem. Int. J. Exp. Cell. Physiol. Biochem. Pharmacol. 51 (1), 11-30. doi: 10.1159/000495157
Zhang, H., and Fan, Q. (2015). MicroRNA-205 inhibits the proliferation and invasion of breast cancer by regulating AMOT expression. Oncol. Rep. 34 (4), 2163-2170. doi: 10.3892/or.2015.4148

Zhang, X. W., Liu, L., Zhang, X. Z., and Bo, P. (2017). Kanglaite inhibits the expression of drug resistance genes through suppressing PVT1 in cisplatinresistant gastric cancer cells. Exp. Ther. Med. 14 (2), 1789-1794. doi: 10.3892/ etm.2017.4650

Zhang, P., Meng, X., Tang, X., Ren, L., and Liang, J. (2019). The effect of a coix seed oil injection on cancer pain relief. Supp. Care Cancer 27 (2), 461-465. doi: $10.1007 / \mathrm{s} 00520-018-4313-\mathrm{z}$

Conflict of Interest: The authors declare that the research was conducted in the absence of any commercial or financial relationships that could be construed as a potential conflict of interest.

Copyright $\odot 2020$ Fang, Jiang, Chen, Huang, Tian, Zhou, Nagle and Zhang. This is an open-access article distributed under the terms of the Creative Commons Attribution License (CC BY). The use, distribution or reproduction in other forums is permitted, provided the original author(s) and the copyright owner(s) are credited and that the original publication in this journal is cited, in accordance with accepted academic practice. No use, distribution or reproduction is permitted which does not comply with these terms. 